\title{
Cherenkov Telescope Ring - An Idea for World Wide Moni- toring of the VHE Sky
}

\author{
Tim Ruhe ${ }^{1, *}$, Dominik Elsässer ${ }^{1, * *}$, Wolfgang Rhode $^{1}$, Maximilian Nöthe ${ }^{1}$, and Kai Brügge ${ }^{1}$ \\ ${ }^{1}$ TU Dortmund University, Dortmund, Germany
}

\begin{abstract}
With very high energy (VHE) gamma-ray astronomy becoming a mature branch of observational astrophysics, and the multi-messenger sky being opened up by neutrino observatories and gravitational wave detectors, we here propose to set up a ring of imaging air Cherenkov telescopes - the Cherenkov Telescope Ring (CTR). The aim of this proposed project would be to facilitate continuous monitoring of sources on the VHE- and multi-messenger sky with minimal time delays and with high sensitivity. Development time and construction cost could be kept comparatively low by both including existing facilities into the monitoring effort, and by relying on substantial previous expertise gained in the community on the road towards the Cherenkov Telescope Array (CTA). This way, the Ring could prove to be a highly efficient facility greatly enhancing the science prospects for future ground-based high-energy astrophysics.
\end{abstract}

\section{Introduction}

Over the preceding quarter of a century, Cherenkov astronomy evolved from a pioneering technology to a well-established method to unveil the existence and energy budget of high energy gamma-ray sources. The findings obtained with Cherenkov telescopes are essential to uncover the astrophysical properties of Galactic and extragalactic gamma-ray sources, important for the understanding of the cosmological past of our Universe and occasionally they even yield unique information for questions from the field of particle physics [1].

To exploit the maximum possible information, Cherenkov measurements are combined with other astronomical observations to achieve a multi - wavelength picture, ranging from radio to the gamma regime. Occasionally information from other messengers, such as neutrinos or gravitational waves may further complete this picture. The comparably small and with energy even rapidly decreasing high-energy gamma-ray flux, however, mandates long Cherenkov observation times per source. Therefore, by performing measurements of single sources, up to now only a part of the sky was covered by Cherenkov observations at a limited sensitivity level. And while there have been dedicated and scientifically highly valuable very high-energy gamma-ray surveys conducted e.g. by the H.E.S.S. collaboration [2, 3], those do not constrain transients in the same sky region happening after the survey. To investigate a multitude of sources in the northern and southern sky the Cherenkov Telescope Array (CTA) [4] is presently planned and set up at two locations.

\footnotetext{
*e-mail: tim.ruhe@tu-dortmund.de

**e-mail: dominik.elsaesser@tu-dortmund.de
} 
Already the first swath of detections of Cherenkov sources other than the Crab nebula showed a possibly high variability of the sources. The temporal information has since been proven to be essential for the understanding of the above-mentioned scientific field. The possible observation time for a specific source is, however, limited by geographical and astronomical conditions, not to mention the limitations due to the weather and other atmospheric conditions. Already now, existing telescopes cooperate in observation campaigns of dedicated objects and for predefined occasions, in order to extend the time for continuous observations. Complete time-surveys of objects in the high-energy gamma sky are, however, not straightforward. Therefore, we propose to set up a ring of telescopes around the globe with an individual sensitivity of at least ten percent of the Crab flux in the energy range above a few hundred $\mathrm{GeV}$. This ring might include existing telescopes, either in the form of a legacy use, or via dedication of observation time. Furthermore, it will have to be completed with new instruments designed for robotic operations.

\section{The Physics case for a Cherenkov Telescope Ring}

The key science drivers that motivate the construction of CTR can be grouped into two categories:

1. Rapid follow-up observations covering a large fraction of the sky.

2. uasi-continuous monitoring of key source populations at unprecedented sensitivity.

With regard to the follow-up science program, CTR is as a natural reaction to the dawn of the era of multi-messenger astronomy. The LAT instrument on the Fermi satellite [5] has for the first time enabled a quasi-continuous monitoring of the gamma-ray sky at $\mathrm{GeV}$ energies. Extending the covered energy range into the $\mathrm{TeV}$ domain and to vastly larger effective areas with the facilities H.E.S.S., MAGIC, VERITAS, MILAGRO and HAWC [2, 6-9] has in many cases proven highly valuable to cover the shortest variability timescales and to open direct windows to the physical processes accelerating particles to very high energies, as evident by the discovery of variability on time scales probably smaller than the light travel time across the event horizon in the case of IC310 [10].

While CTA and installations like HAWC and LHAASO [11] alone will be well suited to this task for the swath of the sky covered by these sites at any given point in time, the highly diverse timescales and lifetimes of AGN flares mandate instantaneous coverage of a dominant portion of the sky and the ability to provide continuous follow-up. Both of these features would be enabled by CTR. Complementing this coverage at even higher energies (albeit with lower statistics), the FACT telescope [12] and the HAWC experiment [13] have started to regularly provide alerts for extreme flares. Broadening our scope to include other messengers, the recent highly successful campaign to follow-up on an extremely high-energy neutrino detected by IceCube and spatially coincident with the blazar TXS 0506+056 has led to the establishment of this object as a VHE source, thus providing strong initial evidence for blazars as a significant source population on the neutrino sky [14]. It is of utmost importance to now establish a much higher number of such cases of coincident detection (or non-detection) in follow-up observations of neutrino events where no previously known VHE source is spatially coincident, to move from single cases towards probing a potential source population. The case of TXS 0506+056 again highlights that the best chances to achieve this in the multi-messenger context may not solely lie in the nearby $\mathrm{TeV}$-bright population of blazars. It is highly encouraging to see that a population of such sources could be readily probed at sensitivities of order ten percent of the Crab flux. 
In addition to the fundamental questions regarding the cosmic accelerators and the origin of the IceCube detected neutrino flux, gravitational wave astronomy has very recently opened up a completely new and exciting window to the Universe.

Photon emission with energies larger than $\approx \mathrm{GeV}$ is only anticipated for those cases of neutron star mergers [15] where a jet is coincidentally launched parallel to our line of sight. The projected sensitivity of the LIGO/VIRGO system, however, is such that is does not seem far-fetched to receive enough alerts to make this statistically happen on year-timescales. In case of a positive detection, substantial knowledge about the contribution of mergers to the high- and very-high-energy Universe can be expected, and potentially even insights on exotic processes at extremely high densities and energies. It is worth noting that in the case of GW170817, the source position rapidly became known with sufficient precision to also allow for ground-based follow-up observations [16]. For existing Cherenkov-facilities, however, horizon constraints were forbidding in this case.

A very recent detection of a GRB by MAGIC [17] shows a substantial discovery space at energies above $300 \mathrm{GeV}$.

Finally, CTR will also open the possibility of follow-ups on a vastly larger number of presumably lower energy triggers like fast radio bursts and magnetar eruptions to conclusively establish very high energy fluxes or upper limits on these elusive phenomena. In the same way as the setup of CTR will provide high chances of suitable conditions for following on trigger events, the Ring will also -for the first time- provide the scientific community with the possibility of nearly continuous monitoring of established sources at almost unlimited timescales. While experiments like FACT and Fermi-LAT have achieved this key capability at low sensitivity and effective area, CTR will be able to provide coverage with sufficient sensitivity to monitor the VHE detected population of flat-spectrum radio quasars (FSRQs). This capability will not only drastically enhance the chances of detecting flaring episodes of this source class and in turn launch multi-wavelength campaigns on these events. It will also facilitate the generation of time-dependent spectral energy distributions. From these studies one does not only expect fundamental insights down to the day-scale of the blazar-flare power spectrum, and into the energy budget and inner jet dynamics of the source population. One could also frame the hadronic contribution to the luminosity, and in turn make physical contact with the measured cosmic high energy neutrino intensity. It is important to note that while the VHE-detected FSRQs will be important monitoring candidates, CTR will also allow for the inclusion of distant but luminous BL Lac objects, like the aforementioned TXS $0506+056$ and more nearby radio galaxies like M87, in the monitoring campaign.

\section{Implementation}

To attain the physical aims, a 24-hour coverage telescope net with a sensitivity of roughly ten percent of the Crab flux above a few hundred GeVs is required. It is intended to reach this goal with minimum additional funding needs within an international collaboration. This would imply that:

- At least parts of the existing capabilities at H.E.S.S., MAGIC, and VERITAS should be used for CTR. Funding would be needed for the maintenance of the instruments and specific measures for the automatization of data taking. This should also include further installations at TAIGA, a site ideally suited to be included into the Ring. Once the instruments are working robotically, they can be maintained by with minimal additional costs.

- Scientific cooperation between CTA and CTR would be mutually beneficial and could greatly enhance multi-messenger campaigns. 


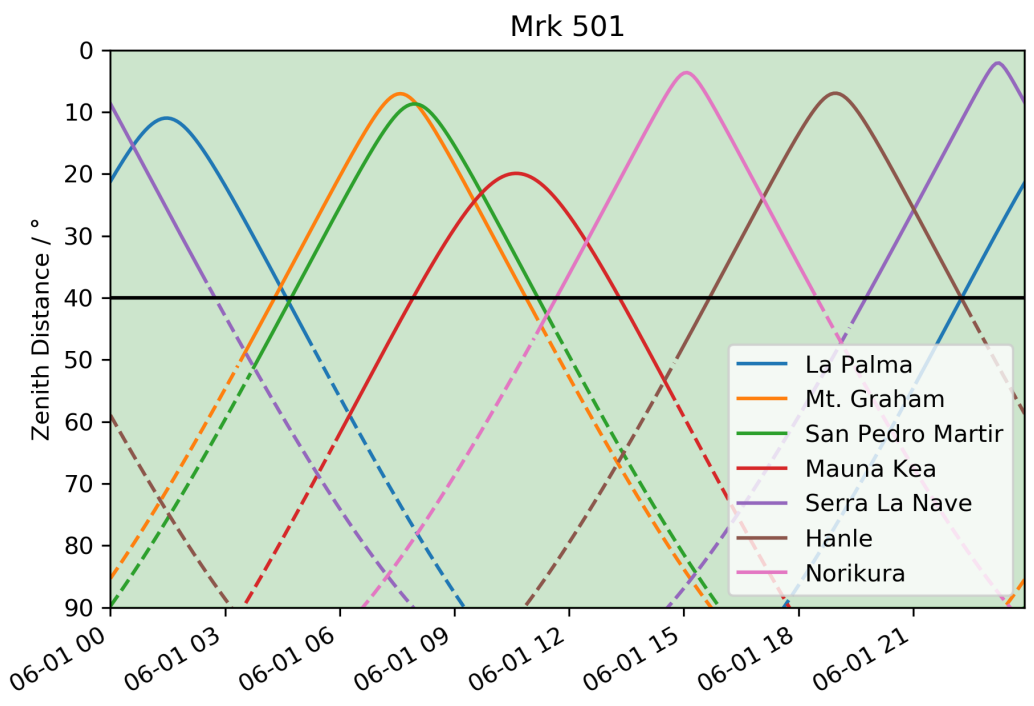

Figure 1. Fiducial visibility plot for Markarian 501 under the assumption of operating a telescope ring like the CTR proposed here, with telescopes installed at a number of well explored sites. The nearly continous observation and monitoring capability of such an installation is readily visible.

- In a first phase, at least two new facilities would be set up, ideally using already developed sites in Russia (TAIGA) and in Australia. The design of these telescopes could be derived as closely as possible from the CTA Medium Size Telescopes (MSTs) [18] ( $\approx 90 \mathrm{~m}^{2}$ mirror area) and modern approaches to camera design conducted in FACT and CTA. Including counting house and computing devices, we envision the total costs per telescope to be on the order 500.000 Euros, and construction times of about two years. A certainly bold, but not unattainable goal, that would bring the new facilites into operation while e.g. H.E.S.S. and MAGIC are still fully running.

- In a second phase, the telescope ring could be complemented by a second set of telescopes of the same type to account for the outage of any single telescope due to the weather conditions or technical problems.

It is further intended to operate the (new) telescopes with resource optimized data analyzing, propagation and storage techniques as presently developed e.g. for the FACT telescope and with a minimal impact for the environment (as "green telescopes").

Acknowledgement: Part of the work on this paper has been supported by Deutsche Forschungsgemeinschaft (DFG) within the Collaborative Research Center SFB 876 Providing Information by ResourceConstrained Analysis, project C3.

\section{References}

[1] A. De Angelis, M. Mallamaci, European Physical Journal Plus 133, 324 (2018), 1805.05642

[2] A. Santangelo, H.E.S.S. Collaboration, The TeV sky with HESS: A review, in Frontier Objects in Astrophysics and Particle Physics, edited by F. Giovannelli, G. Mannocchi (2007), p. 285 
[3] H. E. S. S. Collaboration, AAP 612, A1 (2018), 1804 . 02432

[4] T.C. Consortium, Science with the Cherenkov Telescope Array (World Scientific Publishing Co, 2019), ISBN 981327008X

[5] W.B. Atwood et al., The Astrophysical Journal 697, 1071 (2009), 0902 . 1089

[6] J. Aleksić et al., Astroparticle Physics 72, 76 (2016), 1409. 5594

[7] R. Mukherjee, VERITAS Collaboration, Advances in Space Research 62, 2828 (2018), 1809.10285

[8] A.A. Abdo et al., APJ 664, L91 (2007)

[9] A.U. Abeysekara et al., APJ 841, 100 (2017), 1703.06968

[10] J. Aleksić et al., Science 346, 1080 (2014)

[11] Z. Yao, M. Zha, Z. Cao, H. He et al., 31st ICRC (2009)

[12] M. Noethe et al., PoS ICRC2017, 791 (2018)

[13] S. Westerhoff, Advances in Space Research 53, 1492 (2014), cosmic Ray Origins: Viktor Hess Centennial Anniversary

[14] M. Aartsen et al., Science 361 (2018)

[15] B.P. Abbott et al. (LIGO Scientific Collaboration and Virgo Collaboration), Phys. Rev. Lett. 119, 161101 (2017)

[16] B.P. Abbott et al., The Astrophysical Journal 848, L12 (2017), 1710.05833

[17] R. Mirzoyan, The Astronomer's Telegram 12390 (2019)

[18] G. Pühlhofer, The medium size telescopes of the Cherenkov telescope array, in 6th International Symposium on High Energy Gamma-Ray Astronomy (2017), Vol. 1792 of American Institute of Physics Conference Series, p. 080002, 1610.02899 